\title{
INTRA-ARTERIAL ULTRASONIC IMAGING FOR RECANALIZATION BY SPARK EROSION
}

\author{
N. Bom, C. J. Slager, F. C. van Egmond, C. T. Lancée and P. W. Serruys \\ Thoraxcenter, Erasmus University Rotterdam and University Hospital, Rotterdam-Dijkzigt; Interuniversity \\ Cardiology Institute of the Netherlands, Rotterdam, The Netherlands \\ (Received 8 July 1987; in final form 26 October 1987)
}

\begin{abstract}
Presently several new methods are being developed to recanalize obstructed arteries during catheterization. Intra-arterial high frequency ultrasonic imaging may be used as a guidance for these new techniques. Spark erosion is a new obstruction removal technology. Experiments have shown that this method can be applied in a selective way. An ultrasonic intra-arterial imaging system allows for the proper indication of the spark erosion catheter relative to the obstruction. The first in vitro results of this study illustrate that integration of catheter tip imaging and spark erosion is possible.
\end{abstract}

Key Words: Intra-arterial ultrasound, Recanalization, Miniature transducers.

\section{INTRODUCTION}

New ultrasonic imaging transducers are being developed for application inside the human body. For imaging at close range, high frequencies can be allowed and transducer size can be small. Little has been reported so far on very small transducers capable of imaging from within the human arteries. Nevertheless there exists a major clinical problem that might be solved if a high-quality intra-arterial ultrasonic imaging device were available.

Presently medical treatment for severe obstruction of the coronary arteries is the well known by-pass surgical procedure. A less traumatic recanalization method, introduced by Gruentzig et al. (1979) is the percutaneously applicable catheter balloon dilatation technique. With this method, the obstruction is stretched, not removed, by a small balloon that is gradually inflated. In approximately one third of the treated lesions restenosis occurs.

In the medical world many research efforts are directed towards the development of new techniques for complete removal of the obstruction during cardiac catheterization. Complete removal of the obstruction might reduce the occurrence of restenosis. Such techniques should in addition allow treatment of total or partial obstructions not accessible by the current balloon dilatation techniques. In $5-10 \%$ of

Financial support by the Netherlands Heart Foundation (Grant No. 84.073) is hereby gratefully acknowledged. the treated cases the dilatation procedure is unsuccessful. Dilatation cannot be achieved if the balloon cannot pass the obstruction. In some other cases, notwithstanding the complete procedure being performed no dilatation results.

New methods currently being studied include a rotating abrasive tip as suggested by Hansen et al. (1986); an atherectomy catheter tip method by Simpson et al. (1986) and, for instance, a "hot tip" method as described by Sanborn et al. (1984). Also direct laser application is considered for desobstruction of arteries (Choy et al., 1982; Cross et al., 1986).

One of the main problems of such tissue removal techniques is to prevent arterial perforation. Arterial curvature and the eccentricity of the obstructions in relation to the arterial wall require proper steering of the catheter tip. When, for instance, a glass fiber catheter is guided through an eccentric remaining arterial lumen and laser energy being circumferentially applied to burn away the obstruction, the eccentricity introduces the risk of arterial wall perforation.

For optimal use, the new methods should be made either selective, i.e. normal wall tissue should not be affected by the removal technique, or guided by precise knowledge of the localization and geometry of the arterial obstruction. Intra-arterial echo imaging provides this information. This paper describes the technical aspects and first results of in vitro experiments with high frequency intra-arterial echo imaging integrated with a new recanalization method 
- spark erosion-(Slager et al., 1985) on the tip of a catheter. The described method is aimed in particular at application in cardiology.

\section{Early transducers for use inside the human body}

Already in 1960 Cieszynski $(1960,1961)$ obtained echoes from within the heart with a single catheter-mounted transducer introduced via the jugular vein in dogs. After their first initial report in 1963, Omoto et al. (1963) and Kimoto et al. (1964) published their experiences with an intravenous probe. The carrier of the probe consisted of a stainless steel tube with a $1.2 \mathrm{~mm}$ outside diameter and a wall thickness of $.2 \mathrm{~mm}$. Tomograms were obtained by rotation and withdrawal of the probe. Since transducer displacement was necessarily slow, tomograms were obtained using E.C.G. triggered echo acquisition.

In the meantime real-time imaging of two-dimensional cross-sections emerged. Carleton and Clark (1968) described a catheter-mounted omnidirectionally operating single element. Eggleton et al. (1970) described a rotating catheter system with four elements spaced $90^{\circ}$ apart.

Our laboratory developed the first real-time intracardiac scanner (Bom et al., 1972; Bom, 1973). A 32-element circular array with an outer diameter of $3.2 \mathrm{~mm}$ mounted at the tip of a No. 9 French catheter was constructed. Although the frame-rate (over 100 $\mathrm{s}^{-1}$ ) no longer imposed any timing limitations, we experienced two major complications during in vivo experiments in pigs. Excessive motion in the left ventricular cavity and strong grating lobes. The net result was too ambiguous to be of much clinical value.

\section{Spark erosion}

As reported by Slager et al. (1985) spark erosion can be used to evaporate atherosclerotic plaques. The technique was studied in specimen of human aorta obtained at autopsy. It works well on fatty and fibrous tissue. The method is less suited for removal of purely calcified areas. It has been shown that optimization of the method is possible in order to obtain tissue evaporation combined with diminished side effects such as dehydration and coagulation. Histology showed very little thermal damage of the adjacent tissue zones. Similar to the nonselective laser fiber desobstruction method, spark erosion based on a single electrode may easily perforate the arterial wall because of the eccentric position of the remaining lumen. The eccentric composition of an occlusion in a coronary artery obtained at autopsy is shown in Fig. 1 , panel A.

The results of spark erosion recanalization in the specimen shown in panel $\mathrm{A}$, is demonstrated in panel
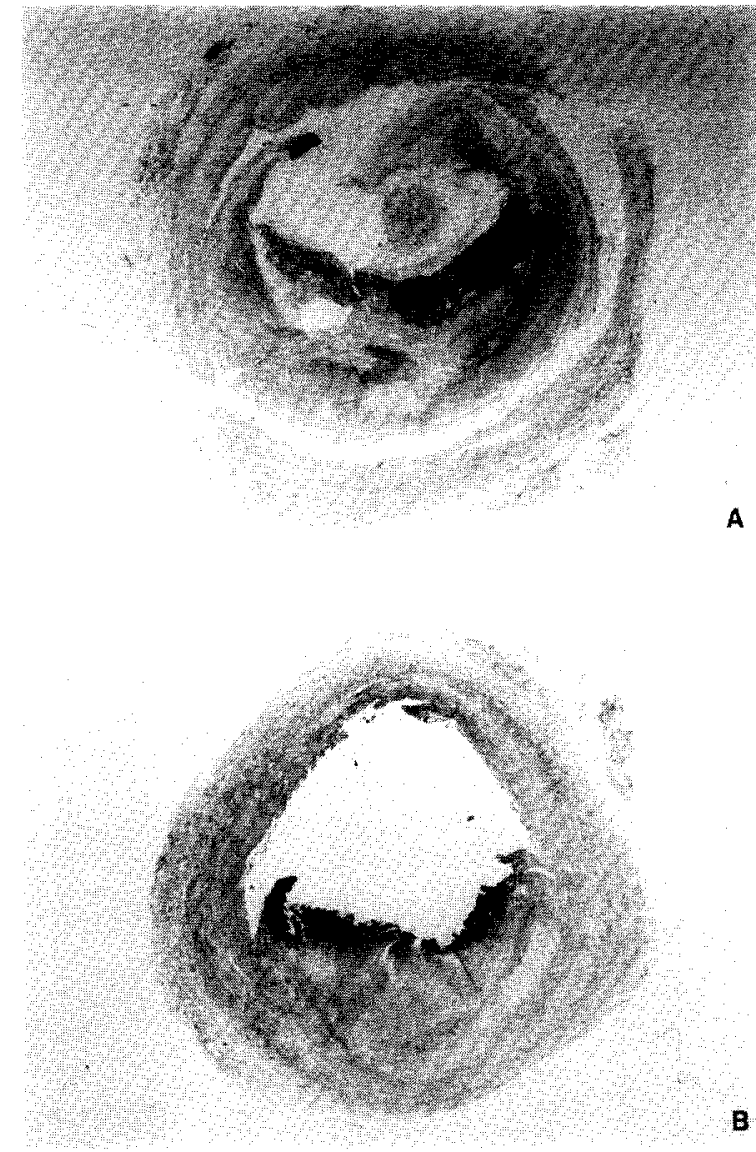

Fig. 1. Cross section (A) of a severely obstructed coronary artery showing eccentric geometry of the remaining lumen. After locally applied erosion, the lumen is eccentrically enlarged (B).

B of Fig. 1. In this example, the lumen was enlarged by use of a tubelike electrode. Spark erosion can be made steerable when more than one electrode is incorporated. Integration in a catheter tip of three electrodes together with the ultrasonic device for imaging of the obstruction was the basis for the first prototype as described hereafter (Slager, 1987).

\section{DESIGN CONSIDERATIONS}

In order to estimate a practical catheter diameter size, it is necessary to have knowledge of the internal coronary artery diameter in normal adults. McAlpin et al. (1973) indicate that the lumen diameter of the right coronary artery ranges from $3.2 \pm 0.6 \mathrm{~mm}$ (proximal) to $2.7 \pm 0.7 \mathrm{~mm}$ (distal). The main left coronary artery lumen diameter was measured to be $4.0 \pm 0.7 \mathrm{~mm}$. For the left anterior descending artery a range was measured of $3.4 \pm 0.5$ (proximal) to 1.9 \pm 0.3 (distal) and for the circumflex of $3.0 \pm 0.7$ (proximal). From this material it was concluded that in a first approach a $2 \mathrm{~mm}$ outer diameter catheter would be sufficiently small. 


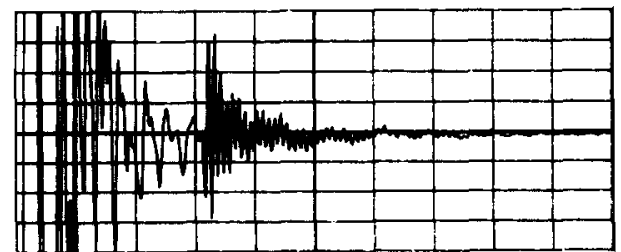

Ch A

$10 \mathrm{mV} / \mathrm{div}$

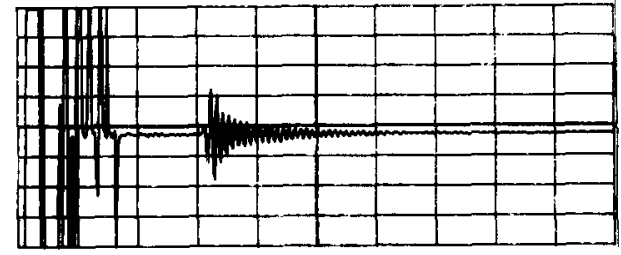

Ch B

$20 \mathrm{mV} / \mathrm{div}$

$T /$ div $5 \mu s$

Fig. 2. The excitation pulse transient effect and reflected pulse from a wire target. Unprocessed signal (A) and averaged, background-corrected $(\mathrm{B})$.

The previously described 32-element cylindrical catheter tip transducer as developed in our laboratory (Bom et al., 1972) is too large with a $3.2 \mathrm{~mm}$ diameter. Diminishing its size to an outer diameter of $2 \mathrm{~mm}$ would be technologically difficult and would require an integrated circuit design for multiplexing transmit and receive signals. The main reason not to follow this course, was the expected transmission pulse transient effect masking the nearby structure echoes. We therefore opted for an approximately $20 \mathrm{MHz}$ single element construction in combination with an acoustic mirror. The diameter of the transducer element was selected to be $1 \mathrm{~mm}$, using conventional technology. In a first set-up this element was provided with air backing and mounted onto a metal bar for study of sensitivity, working frequency, dead zone, echo pulse length and beam characteristics.

As shown in Fig. 2, channel A, the dead zone of

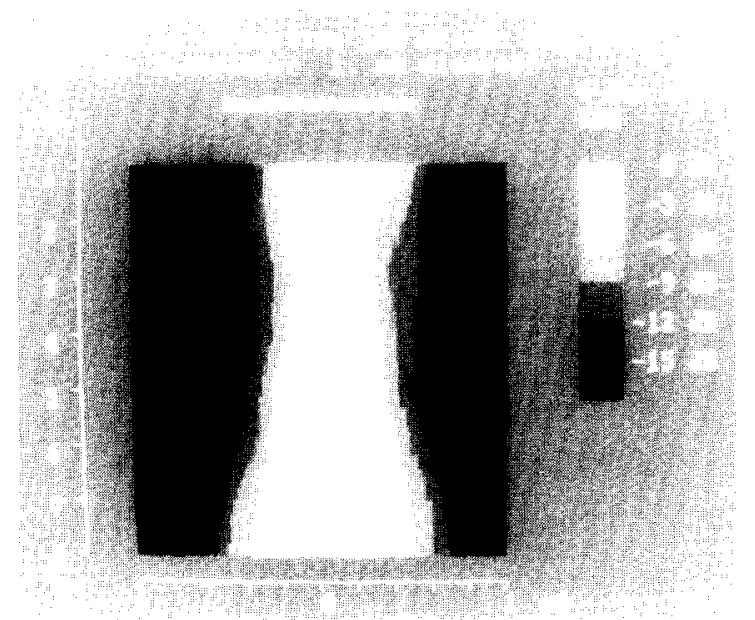

Fig. 3. Beam profile plotted as function of depth for the 1 $\mathrm{mm}$ diameter $18.3 \mathrm{MHz}$ transducer.

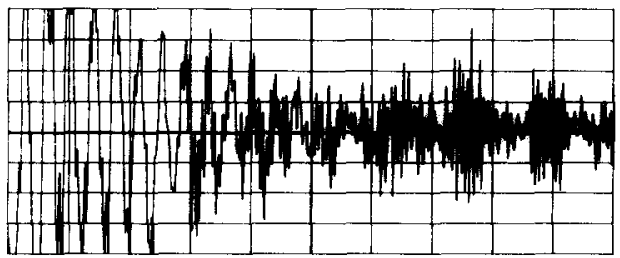

Ch A

$10 \mathrm{mV} / \mathrm{div}$

GLASS

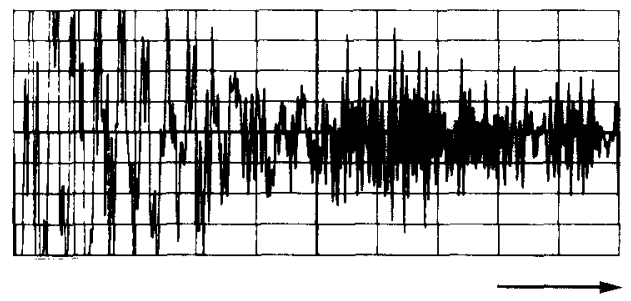

Ch B

$10 \mathrm{mV} / \mathrm{div}$

METAL

T/div .5us

Fig. 4. Pulse transmission and echo reception via a glass mirror (A) and a metal mirror (B).

the unprocessed signal showing the reflection of a 100 $\mu \mathrm{m}$ wire, approximates $1.8 \mu \mathrm{s}$. Averaging ten pulse sequences and subtraction of background improves results drastically, as shown in channel B. For the strong reflecting wire no amplification was necessary in this case. The beam plot of the transducer is shown in Fig. 3.

As a next step the element was mounted in a 2.2 $\mathrm{mm}$ diameter rod and tested in combination with several mirrors. These mirrors were made of different, readily available materials. Reverberation was shown to be smaller with a glass than in a metal mirror. Comparison of the use of a metal and a glass mirror is shown with typical results in Fig. 4.

The beam plot of this assembly with a glass mirror is shown in Fig. 5.

A photograph of the test set is shown in Fig. 6. It represents the transducer assembly that can be step-

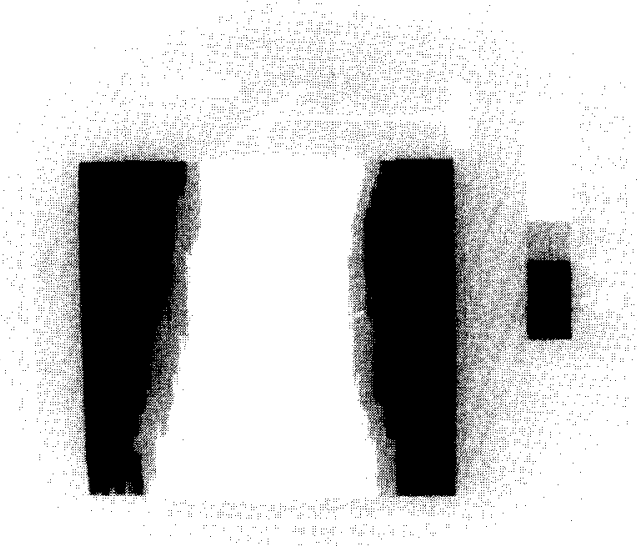

Fig. 5. Beam profile plotted as function of depth for the 1 $\mathrm{mm}$ diameter $19.3 \mathrm{MHz}$ transducer with glass mirror. 


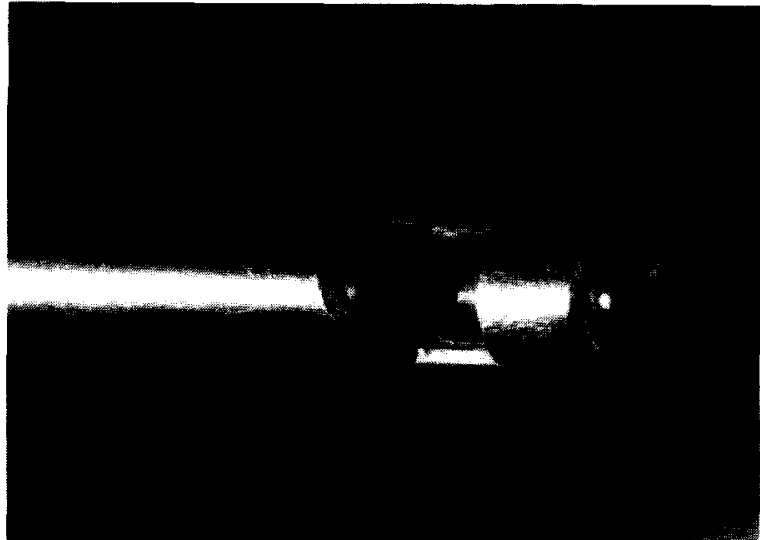

Fig. 6. Fixed transducer assembly driven by a steppingmotor as used for rotational cross-sectional imaging.

rotated and is equipped with angle coding for display purposes.

Experience gained with these preliminary trials led to our decision to design a mechanically rotating catheter tip device that would provide cross-sectional two-dimensional images. Single beam A-mode display resulting from only one or a few forward looking acoustic elements at the tip was regarded as too difficult to interpret.

Spark erosion electrodes must be positioned necessarily at the very tip of the catheter. The cross-sectional imaging plane and the spark erosion plane should preferably be as close together as possible.

\section{RESULTS}

In Fig. 7, a schematic drawing of the echo/recanalization catheter tip is shown. The outer diameter is $2 \mathrm{~mm}$. The mirror (4) is mounted at the end of a flexible wire and can be rotated. The piezoelectric element (5) is positioned over an airbacking for optimal sensitivity. The tip consists of three mutually isolated electrodes. The three electrode wires (2) form an open cage for the echo signals and support the catheter tip. The three electrodes cause the cage echoes in some of the described results. The trans-

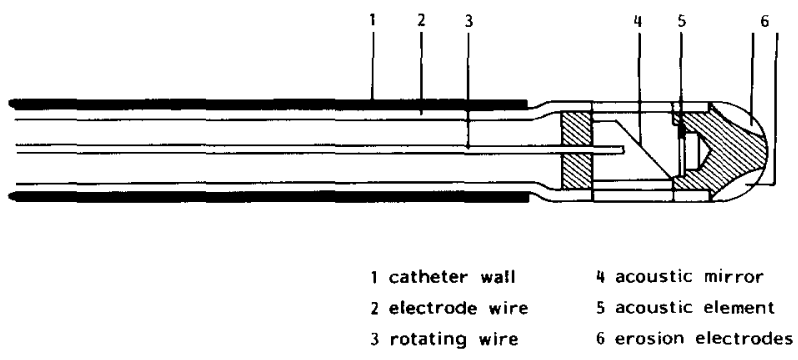

Fig. 7. Schematic drawing of the echo/recanalization catheter tip. Outer diameter is $2 \mathrm{~mm}$.

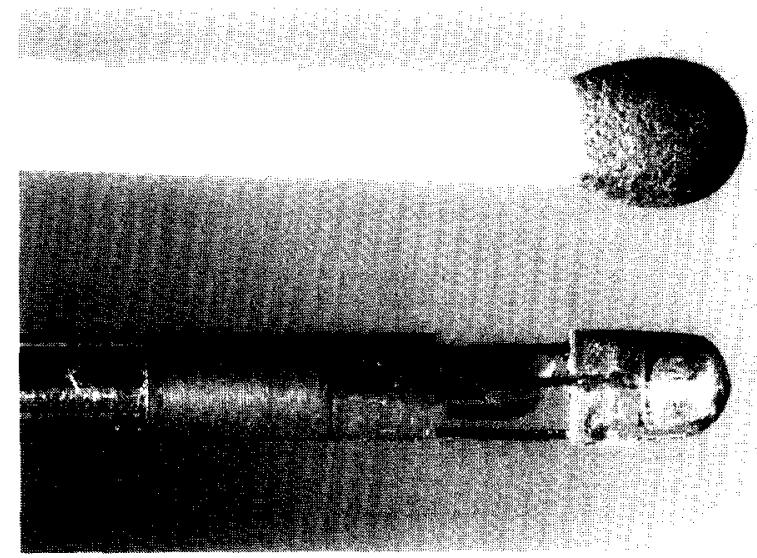

Fig. 8. First prototype for in vitro experiments. This prototype corresponds to the schematic drawing of Fig. 7.

ducer wires are glued onto two of the isolated spark erosion electrodes.

A photograph of a first prototype, with a rigid steel rod instead of a flexible catheter, designed for in vitro measurements, is shown in Fig. 8. An ultrasound intra-arterial image obtained from a specimen of the carotid artery is shown in Fig. 9. As a coupling liquid water was used. The diameter of the lumen of
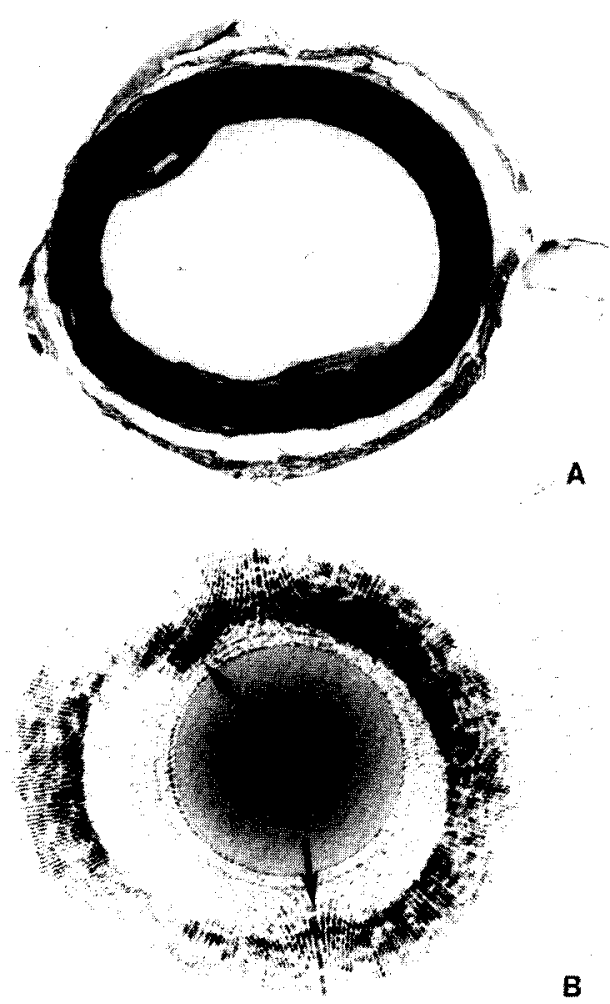

B

Fig. 9. First imaging results illustrated in an arterial specimen (A) and the corresponding intra-arterial echo image (B) as obtained with the transducer assembly shown in Fig. 6. 


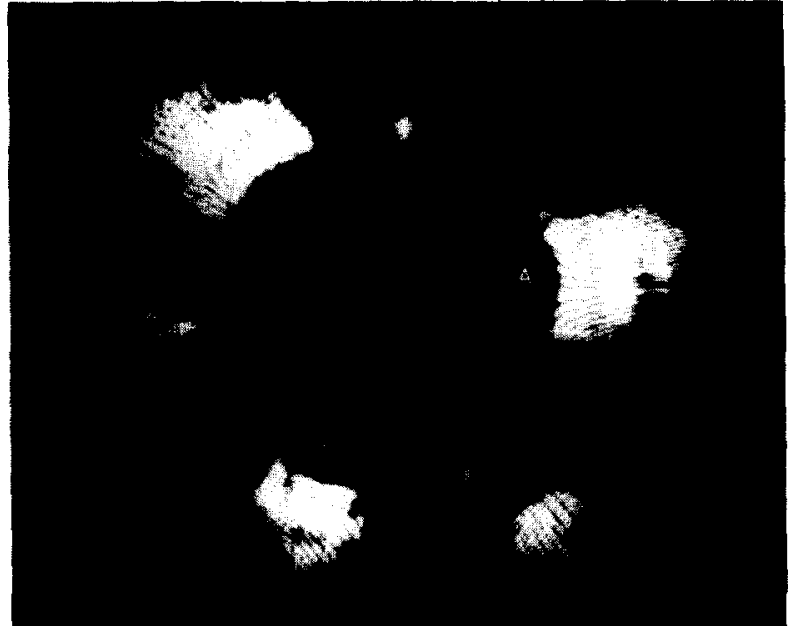

Fig. 10. First image obtained in vitro using the catheter shown in Fig. 8. On this image only the very strong echoes from the cage construction (A) and a plaque (B) are displayed.

this specimen approximates $5 \mathrm{~mm}$. Inner and outer sides of the arterial wall are clearly visible. The two plaque areas are clearly visible on the anatomic cross section as well as on the echo image. This image has been obtained with the in vitro set-up with the device shown in Fig. 6. A first result from an in vitro image as obtained with the catheter is shown in Fig. 10. In this image the three cage echoes (A) and the echo of the calcified plaque (B) are clearly visible. These images show the possibilities of intra-arterial structure localization. Further studies must teach us the limitations caused by the cage echoes and the possible design modifications.

\section{CONCLUSION}

New recanalization methods such as spark erosion require steering in order to cope with the eccentricity of the occlusion. A possible method of guidance is ultrasonic imaging with a single miniature high frequency element. With a $20 \mathrm{MHz}$ catheter tip prototype with a $1 \mathrm{~mm}$ diameter single element and rotating mirror technique first intra-arterial crosssectional echo images were obtained in vitro. Our experiments show that it is technologically possible to integrate the imaging device and the spark erosion electrodes in a catheter tip with a diameter of only 2 $\mathrm{mm}$. In vitro tests lead us to believe that this new method has great potential.
Acknowledgements-The construction of the catheter tip has been a cooperation of the Central Research Workshop of the Erasmus University Rotterdam, with the assistance of Messrs. R. Niesing, A. H. den Ouden, J. Bos and L. Bekkering, and the Productcenter of T.N.O.-Delft, where Messrs. Plukaard en Van Soest actually made the first prototype.

We furthermore acknowledge the contribution of the Audio Visual Center of the Erasmus University for the illustrations in this article.

\section{REFERENCES}

Bom N., Lancée C. T. and Van Egmond F. C. (1972) An ultrasonic intracardiac scanner. Ultrasonics 10, 72-76.

Bom N. (1973) Apparatus for ultrasonically examining a hollow organ. Patent specification 1,402,192, filed February 22.

Carleton R. A. and Clark J. G. (1968) Measurement of left ventricular diameter in the dog by cardiac catheterization. Validation and physiologic meaningfulness of an ultrasonic technique. Circ. Res. 22, 545-548.

Choy D. S. J., Stertzer S., Rotterdam H. Z. and Bruno M. S. (1982) Laser coronary angioplasty: experience with 9 cadaver hearts. Am. J. Cardiol. 50, 1209-1211.

Cieszynski T. (1960) Intracardiac method for the investigation of structure of the heart with the aid of ultrasonics. Arch. Immun. Ter. Dosw. 8, 551-557.

Cieszynski T. (1961) Intracardiac method of ultrasound heartstructure investigation. Polsk. Przeglad. Chirurg. 33, 1071.

Cross F. W., Bowker T. J. and Bown S. G. (1986) Contact sapphire tip angioplasty with a pulsed Nd-YAG laser. Lasers Med. Sci. 1, 311.

Eggleton R. C., Townsend C., Herrick J., Templeton G. and Mitchell J. H. (1970) Ultrasonic visualization of left ventricular dy. namics. Ultrasonics 17, 143-153.

Gruentzig A. R., Senning A. and Siegenthaler W. E. (1979) Nonoperative dilatation of coronary artery stenosis: percutaneous transluminal angioplasty. $N$. Engl. J. Med. 301, 61-68.

Hansen D. D., Hall M., Intlekofer M. J., Auth D. and Ritchie J. L. (1986) In vivo rotational angioplasty in atherosclerotic rabbits; comparison of angioscopy and angiography. Circulation 74, Supp. II: 362 (abstract).

Kimoto S., Omoto R., Tsunemoto M., Muroi T., Atsumi K. and Uchida R. (1964) Ultrasonic tomography of the liver and detection of heart atrial septal defect with the aid of ultrasonic intravenous probes. Ultrasonics 2, 82-86.

McAlpin R. N., Abbasi A. S., Grallman J. A. and Eber L. (1973) Human coronary artery size during life. Radiology $\mathbf{1 0 8}$ 567-573.

Omoto R., Atsumi K., Suma K., Toyoda T., Sakurai Y., Muroi T., Fujimori Y., Idezuki Y., Tsunemoto M., Sugiwura M. and Saegusa M. (1963) Ultrasonic intravenous sonde-2nd report. Med. Ultrason. (Jpn) 1, 11.

Sanborn T. A., Faxon D. P., Haudenschild C. C. and Ryan T. J. (1984) Laser radiation of atherosclerotic lesions: decreased incidence of vessel perforation with a fiberoptic laser heated metallic tip (abstr). J. Am. Coll. Cardiol. 3, 490.

Simpson J. B., Johnson D. E., Brader L. J., Gifford H. S., Thapliyal H. V. and Selmon M. R. (1986) Transluminal coronary atherectomy (TCA): results in 21 human cadaver vascular segments. Circulation 74, Supp. II: 202 (abstract).

Slager C. J., Essed C. E., Schuurbiers J. C. H., Bom N., Serruys P. W. and Meester G. T. (1985) Vaporization of atherosclerotic plaques by spark erosion. J. Am. Coll. Cardiol. 5, 1382-1386.

Slager C. J. (1987) Echo/Vonkerosie Recanalisatie Inrichting. Dutch Patent Application No. 8700632 of March 17. 